\title{
Grenzüberschreitende regionale Identität in der Regio TriRhena von Studierenden der Universitäten Basel, Freiburg im Breisgau und Mulhouse
}

\author{
Christine Griebel, Basel
}

Regionale Identität gestaltet sich zum einen in den Sozialbeziehungen der Bewohner einer Region aus, zum anderen in deren Verbundenheit mit dem Raum. Auch über kulturelle oder politische Grenzen hinweg kann sich ein Regionalbewusstsein ausbilden. Die Regio TriRhena ist ein trinationaler Raum mit vielfältigen grenzüberschreitenden Verflechtungen, welche sich günstig auf die Herausbildung einer regionalen Identität über die Grenzen hinweg auswirken können. Mittels einer Befragung unter Studierenden an den Universitäten Basel, Freiburg im Breisgau und Mulhouse wurden die raumbezogene Identifikation der Befragten, ihre sozialen Netzwerke sowie ihr raumrelevantes Verhalten wie z.B. die grenzüberschreitende Mobilität untersucht.

\section{Hintergrund der Studie}

\subsection{Die Trinationale Region am Oberrhein}

Die Region am südlichen Oberrhein zeichnet sich durch die Lage im Grenzgebiet von der Schweiz, Deutschland und Frankreich und durch vielfältige und enge Verflechtungen zwischen den drei Regionen aus. In wirtschaftlicher Hinsicht ist eine lange Tradition von Pendlerbeziehungen über die Grenzen hinweg auszumachen, Unternehmen profitieren von der Lage in der Mitte Europas und vom Abbau von Barrieren im Zuge der europäischen Integration. Historische Verbindungen wie die gemeinsame Zugehörigkeit zum alemannischen Sprach- und Kulturraum existieren, die Geschichte kennt aber auch Verwerfungen, die sich im Elsass mit mehrmaligem Wechsel zwischen deutscher und französischer Staatsangehörigkeit und Sprache besonders drastisch auswirkten.

In den vergangenen Jahrzehnten wurde am Oberrhein auf verschiedenen Ebenen eine grenzüberschreitende Kooperation angeregt und ausgebaut. Dies geschah zum einen europaweit, beispielsweise mit der Einführung der Euro-Währung und dem Schengener Abkommen (dem letzteren ist inzwischen auch die Schweiz beigetreten), so dass der Abbau von Barrieren auch am Oberrhein sichtbar und spürbar wurde. Die Schweiz beteiligt sich zudem an den Interreg-Programmen der Europäischen Union, die gezielt die interregionale Zusammenarbeit fördern. $\mathrm{Zu}$ nennen sind ausserdem die Kooperationen zwischen Kommunen, Kantonen, Landkreisen und weiteren Körperschaften aus allen drei Ländern, die eine Abstimmung in Sachfragen ermöglichen. Dazu gehören der Trinationale Eurodistrict Basel, der Verein RegioTriRhena und die Oberrheinkonferenz sowie im Bereich der Bildung der oberrheinische Hochschulverbund EUCOR mit den Universitäten Basel, Freiburg im Breisgau, Karlsruhe, Strasbourg und Mulhouse. Grenzüberschreitende Zusammenarbeit erfolgt jedoch nicht nur «Top-Down» beziehungsweise von Seiten der politischen Entscheidungsträger und Institutionen, sondern auch «Bottom-Up» aus der Zivilgesellschaft heraus, beispielsweise durch die in den 1970er Jahren entstandenen badisch-elsässischen Bürgerinitiativen, die den Widerstand gegen das damals geplante Atomkraftwerk Wyhl grenzüberschreitend organisierten.

Diese Entwicklungen auf verschiedenen politischen und gesellschaftlichen Ebenen legen die Frage nahe, $\mathrm{ob}$ in der Oberrheinregion die Grenzen auch im Bewusstsein der Bevölkerung verschwinden, ob sich also eine grenzüberschreitende Identität der RegioBewohner herausbildet.

\subsection{Regionale Identität als Forschungsgebiet der Geographie}

Drei Grunddimensionen raumbezogener Identität werden im Theorieansatz von Blotevogel, Heinritz und PopP (1989) unterschieden: Erstens die räumliche Identität, die das Einstellungskonzept, die kognitivemotionale Repräsentation eines Raumes im Bewusstsein eines Individuums oder einer sozialen Gruppe darstellt, zweitens die individuelle räumliche Identifikation, also mentale und affektive Besetzung eines Raumes in der Selbstkonzeption des Individuums, und drittens die soziale räumliche Identifikation, die sich auf die Identitätsbildung von sozialen Systemen bezieht. Diese Konzeption wird von HARD (1987) hinterfragt, der argumentiert, dass sich Identität als Bestandteil sozialer Kommunikation nicht räumlich fixieren lässt. Auch Werlen (1992) betont, dass es problematisch sei, regionale Identitäten in erdräumlichen Kategorien zu lokalisieren und sieht das Erkenntnisinteresse der Sozialgeographie stattdessen darin, die regionalen Bedingungen für soziale Kommunikation zu analysieren. Inzwischen wurden in der Sozialgeographie und den Nachbardisziplinen zahlreiche empirische Studien zur regionalen Identität mit unterschiedlichen Fragestellungen und in verschiedenen Regionen durchgeführt, während die theoretischen Grundsatzdebatten abgeflaut sind (Aschauer 2000; vgl. Fichtner 1988; MÜHLER \& OPP 2004; SCHUHBAUER 1996). 
Regionale Identität wird in der Forschung allerdings nicht nur empirisch-analytisch untersucht, sondern oftmals auch in normativer Hinsicht als Ziel der Regionalplanung beschrieben, da man sich von einer positiven regionalen Identität die Aktivierung endogener Potentiale und die Entfaltung ökonomischer, sozialer und kultureller Aktivitäten erhofft (IPSEN 1994:232).

Zur Erklärung der Entstehung und unterschiedlichen Ausprägung regionaler Identität werden verschiedene Konzepte herangezogen: Auf der Ebene des Individuums ist besonders die Sozialisationshypothese verbreitet, d.h. die Geburt, das Aufwachsen in der Region und die Wohndauer werden als Prädiktor eines starken Regionalbewusstseins angenommen (MüHLER \& OPP 2004: 67; SCHNEIDER et al. 2003: 118). Würden aber nur individuelle, persönliche Faktoren der eigenen Biographie auf die Herausbildung einer regionalen Identifikation des Einzelnen wirken, so könnte man allenfalls von der Gleichzeitigkeit ähnlicher Wahrnehmungen und Gefühle sprechen, aber nicht von einer kollektiven Identität. Eine solche wird durch gruppenspezifische Erfahrungen herausgebildet, durch gemeinsam geteilte Erfahrungen und durch soziale Interaktion. Die Sprache als Medium für Kommunikation und soziale Interaktion ermöglicht den Zugang zu Sozialleben und Kultur und bildet damit eine Grundvoraussetzung für die Identitätsbildung. Über die Kultur werden identitätswirksame Symbolsysteme vermittelt, die gemeinsame Werte repräsentieren.

Eine besondere Rolle in der Herausbildung von kollektiver Identität spielen Grenzen und Abgrenzungen: Eine Gesellschaft wird durch ihre scharfe Abgrenzung als innerlich zusammengehörig charakterisiert, und umgekehrt äussert sich die innere Einheit und funktionelle Beziehung zwischen ihren Bestandteilen auch räumlich in der einrahmenden Grenze (SIMMEL 1908: 693). Grenzen sind für den Menschen notwendig, um Raum, Zeit und Gesellschaft zu organisieren. Sie stehen aber auch für die Vielfalt von politischen Systemen, Kultur und sozialer Organisation und erlauben es so, die Vorteile unterschiedlicher Systeme zu erkennen (LeIMGRUBER 2005: 246f.).

Die Anwesenheit von Grenzen wirkt sich auf die Bevölkerung in Grenzregionen aus, so dass GuichoNNET und RAFFESTIN (1974: 149) dieser Bevölkerung eine gewisse Marginalität, Originalität und Ambiguität zuschreiben. Das Ausmass solcher spezifischer Eigenschaften ist davon abhängig, wie stark die Bevölkerung an verschiedenen Räumen, Kulturen und Informationsmedien teilhat und ob die sprachlichen Barrieren gering sind bzw. sich eine Mehrsprachigkeit etabliert hat. Auch Martinez (1994) macht in der Gesellschaft in Grenzregionen, verglichen mit dem Landesinneren, besondere Prozesse und Charakteri- stika aus, bedingt durch das binationale Milieu. Die Bewohner der Grenzregion gehen unterschiedlich mit dieser Situation um, so dass vereinfacht zwei Typen von Grenzlandbewohnern unterschieden werden können (MARTINEZ 1994: 6): National borderlanders mit wenig Kontakt jenseits der Grenze und Gleichgültigkeit gegenüber den Nachbarn sind nicht willens oder fähig, in einer anderen Gesellschaft zu funktionieren. Dagegen haben transnational borderlanders bedeutende Verbindungen zum Nachbarland, sie nutzen die Möglichkeiten, die andere Seite zu besuchen, dort einzukaufen, zu arbeiten, zu studieren oder vorübergehend zu wohnen. In grenzübergreifenden Agglomerationen wie dem Raum Basel unterstreicht die Grenze die Identitäten: die Grenzüberschreitung bleibt trotz der Kontinuität innerhalb eines städtischen Raumes ein Übergang ins «Andere» (REITEL 2007: 6).

Grenzen dienen also nicht nur der Abgrenzung, sondern bieten durch die Auseinandersetzung mit den «Anderen» auch die Chance, Gemeinsamkeiten mit diesen wahrzunehmen (WeIGL 2005). Regionale Identität kann sich so auch über Grenzen hinweg ausbilden.

\section{Methodik}

Die Untersuchung baut auf einer vorgängigen Studie aus dem Jahr 1999 von EdER SANDTNER und SANDTNER (2003) auf, welche die Wahrnehmung der regionalen Identität unter den Bewohnern der Regio TriRhena zum Gegenstand hatte. Die aktuelle Studie von 2009 bezieht sich explizit auf die Studierenden in der Regio TriRhena, da deren zukünftige Rolle z.B. als LehrerInnen, WissenschaftlerInnen oder leitendes Personal in Wirtschaft und Verwaltung eine Meinungsbildner- und Multiplikatorenfunktion einschliesst, und somit von gesamtgesellschaftlicher Bedeutung ist (SCHNEIDERSLIWA et al. 2009). Nicht zuletzt bieten Hochschulkooperationen in Grenzregionen - wie EUCOR - ein Potential für Annäherungsprozesse im Hochschulalltag, von denen Ausstrahlungseffekte auf den Raum insgesamt erwartet werden können (FICHTER-Wolf 2009: 201).

Die Befragung erfolgte über einen Online-Fragebogen in deutscher und französischer Sprache, auf den die Studierenden der Universitäten Basel, Freiburg i. Br. und Mulhouse mittels E-Mail-Verteilern, Flyern und Aushängen verwiesen wurden. Im Mittelpunkt standen Fragen zur Identifikation der Studierenden mit der Region, zur Bekanntheit und Bewertung der interregionalen Zusammenarbeit und zur grenzüberschreitenden Mobilität der Studierenden. Aus den Umfrageergebnissen wurden über statistische Methoden wie Häufigkeitsverteilungen und Kreuztabellierungen das grenzüberschreitende Verhalten der Studierenden und 
ihre Einstellung zur Regio TriRhena in Abhängigkeit von personenbezogenen Variablen ermittelt.

Der Rücklauf der Umfrage betrug 651 gültige Fragebogen aus Basel (bei einer Grundgesamtheit von ca. 11000 Studierenden), 267 aus Freiburg (Grundgesamtheit: ca. 20 000) sowie 395 aus Mulhouse (Grundgesamtheit: ca. 8000 ), was eine gesamte Stichprobengrösse von 1313 Studierenden ergibt. An allen drei Standorten nahmen mit insgesamt 58\% mehr Frauen als Männer an der Befragung teil.

\section{Regionale Identität von Studierenden in der Regio TriRhena}

\subsection{Gemeinsame Sprache als Voraussetzung für soziale Interaktion}

Kommunikation und soziale Interaktion gelten als zentrale Elemente in der Herausbildung einer kulturellen oder regionalen Identität. Also kann die Sprachkompetenz Aufschluss darüber geben, ob und wie stark man lokal verwurzelt ist oder sich mit der grösseren Region verbunden fühlen kann. Am Oberrhein sind mit Schweizerdeutsch, Badisch und Elsässisch in allen drei Teilen der Regio alemannische Dialekte verbreitet, jedoch in unterschiedlicher Ausprägung. Schweizerdeutsch wird von fast allen Basler Studierenden gesprochen und Badisch immerhin von über $40 \%$ der Freiburger Studierenden - viele Studierende kommen auch aus anderen Teilen Deutschlands nach Freiburg. Dagegen ist nur ein Viertel der Studierenden an der Universität Mulhouse des elsässischen Dialekts mächtig. In der Vorgängerstudie von EDER SANDTNER und SANDTNER war dies noch die Hälfte der Befragten. Allerdings wurden in jener Studie alle Bevölkerungsund Altersschichten befragt. Bei der jungen Generation verliert offensichtlich der Dialekt zu Gunsten der Schriftsprache stark an Bedeutung, was auch in anderen Untersuchungen zur Sprachkompetenz im Elsass beobachtet wurde (HARTwEg 2003). Immerhin 60\% der Befragten aus Mulhouse sprechen Hochdeutsch, Französisch wird von rund der Hälfte der Basler und Freiburger Studierenden aktiv gesprochen. Auch wenn die Studierenden damit über gute Sprachkenntnisse verfügen, nimmt die Mehrheit von ihnen die Grenzen in der Regio besonders als sprachliche Grenzen wahr, und nur ein Zehntel bezeichnet die Regio als Raum mit gemeinsamer Sprache.

Durch den Fokus der Untersuchung auf die Zielgruppe der Studierenden konnten in den Ergebnissen gewisse Unterschiede zur Vorgängerstudie von EDER SANDTNER und SANDTNER erwartet werden. In sprachlicher Hinsicht sind genügend hohe Anteile von Studierenden im Sample, welche Sprachkompetenz im Dialekt oder der Sprache des Nachbarlandes haben, was sich positiv auf ein Regionalbewusstsein auswirken könnte. Wenn die Regio TriRhena auch im Bewusstsein der Bevölkerung zusammenwächst, so müsste sich dies bei der vergleichsweise jungen Altersgruppe der Studierenden und zehn Jahre nach der Vorgängerstudie in einer stärker ausgeprägten regionalen Identität äussern. Auch das allgemein höhere Bildungsniveau von Studierenden müsste sich positiv auf eine regionale Identifikation auswirken. Die hohen Erwartungen müssen allerdings etwas relativiert werden, bedenkt man, dass die Universitäten auch viele Studierende aus anderen Landesteilen anziehen, die daher erst wenige Jahre in der Regio wohnhaft sind und demzufolge eine schwächer ausgeprägte Identifikation mit diesem Raum zeigen dürften.

\subsection{Soziale Netzwerke und Mobilität}

Da die regionale Identität unter anderem durch soziale Interaktion geprägt wird, ist die Herausbildung einer regionalen Identität stark von grenzüberschreitenden Sozialkontakten und der Mobilität der Bevölkerung abhängig. Allerdings gibt es unter Studierenden in der Regio TriRhena nur wenige verwandtschaftliche und freundschaftliche Kontakte über die Grenzen hinweg. Über die Hälfte der Befragten aller Regionen pflegt keinerlei freundschaftliche Kontakte jenseits der Grenze. Die meisten Beziehungen über die Grenzen sind nicht sehr intensiv. Nur in Mulhouse geben mit knapp einem Fünftel vergleichsweise viele Studierende an, intensiven Kontakt mit Freunden aus der Regio zu pflegen.

Aus der zehn Jahre zurückliegenden Vergleichsstudie von Eder Sandtner und Sandtner, die auf die breite Bevölkerungsschicht gerichtet war, ging hervor, dass grenzüberschreitende Aktivitäten der RegioBewohner stark ausgeprägt sind. Die damals befragten Regio-Bewohner gaben an, häufig zum Einkaufen und zur Erholung in die benachbarte Regio zu fahren. Die Ergebnisse der aktuellen Untersuchung weisen Parallelen zur Vorgängerstudie auf. Heute sind die grenzüberschreitenden Aktivitäten der Studierenden zum Einkaufen und Essen mässig bis stark ausgeprägt (Tab. 1).

Es ist deutlich, dass Basler Studierende häufiger in Südbaden und dem Elsass einkaufen gehen, was sicherlich dem Lohn- und Preisgefälle zwischen der Schweiz und den Nachbarländern zuzuschreiben ist. Die Nordwestschweiz mit höherem Preisniveau wird eher weniger als Einkaufsdestination genannt. Im Vergleich zu 1999 besuchen die Befragten weniger kulturelle Veranstaltungen in Südbaden und dem Elsass als ein Durchschnitt der gesamten Bevölkerung, für die Nordwestschweiz sind die Werte praktisch gleich. Die kulturellen Angebote der Region Basel scheinen also eine gewisse Anziehungskraft für Freiburger und Mul- 


\begin{tabular}{|c|c|c|c|c|c|c|}
\hline \multirow[b]{2}{*}{$\begin{array}{l}\text { Absicht und Häufigkeit } \\
\text { der Grenzüberquerung }\end{array}$} & \multicolumn{2}{|c|}{ Studierende der Uni Basel } & \multicolumn{2}{|c|}{ Studierende der Uni Freiburg } & \multicolumn{2}{|c|}{ Studierende der Uni Mulhouse } \\
\hline & $\begin{array}{c}\text { Nach } \\
\text { Südbaden } \\
\text { in } \% \\
(\mathrm{n}=414) \\
\end{array}$ & $\begin{array}{l}\text { Ins Elsass } \\
\text { in } \% \\
(n=290)\end{array}$ & $\begin{array}{c}\text { In die Nord- } \\
\text { westschweiz } \\
\text { in \% } \\
(\mathrm{n}=183) \\
\end{array}$ & $\begin{array}{l}\text { Ins Elsass } \\
\text { in } \% \\
(\mathrm{n}=201)\end{array}$ & $\begin{array}{c}\text { In die Nord- } \\
\text { westscheiz } \\
\text { in } \% \\
(\mathrm{n}=270) \\
\end{array}$ & $\begin{array}{c}\text { Nach } \\
\text { Südbaden } \\
\text { in } \% \\
(\mathrm{n}=242) \\
\end{array}$ \\
\hline \multicolumn{7}{|l|}{ Einkaufen } \\
\hline $\begin{array}{l}\text { Mindestens einmal im } \\
\text { Monat }\end{array}$ & 33.3 & 11.7 & 10.4 & 12.4 & 23.8 & 49.2 \\
\hline $\begin{array}{l}\text { Seltener als einmal im } \\
\text { Monat }\end{array}$ & 54.8 & 44.5 & 43.7 & 54.7 & 51.5 & 42.6 \\
\hline Nie & 11.8 & 43.8 & 45.9 & 32.8 & 24.8 & 8.3 \\
\hline \multicolumn{7}{|l|}{ Erholung } \\
\hline $\begin{array}{l}\text { Mindestens einmal im } \\
\text { Monat }\end{array}$ & 16.5 & 14.8 & 7.6 & 7.0 & 25.5 & 35.9 \\
\hline $\begin{array}{l}\text { Seltener als einmal im } \\
\text { Monat }\end{array}$ & 52.7 & 66.2 & 69.9 & 74.6 & 47.8 & 46.7 \\
\hline Nie & 30.4 & 19.0 & 22.4 & 18.4 & 25.2 & 17.4 \\
\hline \multicolumn{7}{|l|}{ Gastronomiebesuch } \\
\hline $\begin{array}{l}\text { Mindestens einmal im } \\
\text { Monat }\end{array}$ & 14.7 & 5.8 & 2.7 & 4.0 & 9.6 & 20.7 \\
\hline $\begin{array}{l}\text { Seltener als einmal im } \\
\text { Monat }\end{array}$ & 51.7 & 53.1 & 32.2 & 43.8 & 25.6 & 41.3 \\
\hline Nie & 33.6 & 41.0 & 65.0 & 52.2 & 64.8 & 38.0 \\
\hline \multicolumn{7}{|l|}{$\begin{array}{l}\text { Kulturelle } \\
\text { Veranstaltungen }\end{array}$} \\
\hline $\begin{array}{l}\text { Mindestens einmal im } \\
\text { Monat }\end{array}$ & 6.9 & 1.0 & 10.4 & 3.0 & 12.2 & 14.9 \\
\hline $\begin{array}{l}\text { Seltener als einmal im } \\
\text { Monat }\end{array}$ & 44.2 & 32.8 & 77.6 & 48.3 & 44.4 & 34.7 \\
\hline $\mathrm{Nie}$ & 48.8 & 66.2 & 12.0 & 48.8 & 43.3 & 50.4 \\
\hline \multicolumn{7}{|l|}{ Studium } \\
\hline $\begin{array}{l}\text { Mindestens einmal im } \\
\text { Monat }\end{array}$ & 1.9 & 1.0 & 7.6 & 2.0 & 7.8 & 8.1 \\
\hline $\begin{array}{l}\text { Seltener als einmal im } \\
\text { Monat }\end{array}$ & 20.5 & 8.3 & 24.6 & 14.9 & 17.8 & 14.9 \\
\hline Nie & 77.5 & 90.7 & 67.8 & 83.1 & 74.4 & 76.0 \\
\hline
\end{tabular}

Tab. 1: Häufigkeit und Absicht von Grenzüberquerungen

Frequency and intention of border crossings

Fréquence et motivations des passages frontaliers

Quelle: Geographisches Institut der Universität Basel, Stadt- Und Regionalforschung; Studierendenbefragung 2009

houser Studierende zu besitzen. In allen drei Städten gaben etwa drei Viertel der Studierenden an, für Freizeitaktivitäten wie Velo fahren, Baden oder Wandern in die angrenzenden Regionen zu fahren, wenn auch in unterschiedlicher Häufigkeit und im Allgemeinen seltener als einmal im Monat. Es fällt auf, dass die grosse Mehrheit der Studierenden nie aus Gründen des Studiums die Grenze überquert. Die Nutzung von Austauschprogrammen für Schüler und Studierende ist in den drei untersuchten Teilregionen sehr unterschiedlich. Basler Studierende nutzen die Möglichkeiten zum universitären Austausch am wenigsten und hatten auch in ihrer Schulzeit kaum an einem Schüleraustausch teilgenommen. Auch in Mulhouse ist die 


\begin{tabular}{|c|c|c|c|c|c|c|}
\hline \multirow{2}{*}{ Selbstsicht } & \multicolumn{2}{|c|}{ Basel } & \multicolumn{2}{|c|}{ Freiburg } & \multicolumn{2}{|c|}{ Mulhouse } \\
\hline & absolut & in \% & absolut & in \% & absolut & in $\%$ \\
\hline Europäer/in & 173 & 26.6 & 116 & 43.4 & 72 & 18.2 \\
\hline $\begin{array}{l}\text { Schweizer/in; Deutsche/r; } \\
\text { Franzose/Französin }\end{array}$ & 318 & 48.8 & 72 & 27.0 & 188 & 47.6 \\
\hline $\begin{array}{l}\text { Nordwestschweizer/in; } \\
\text { Baden-Württemberger/in; } \\
\text { Elsässer/in }\end{array}$ & 36 & 5.5 & 33 & 12.4 & 100 & 25.3 \\
\hline Regiobewohner/in & 24 & 3.7 & 23 & 8.6 & 16 & 4.1 \\
\hline $\begin{array}{l}\text { Anderes (z.B. Weltbürger, } \\
\text { Mensch) }\end{array}$ & 100 & 15.4 & 23 & 8.6 & 19 & 4.8 \\
\hline Gesamt & 651 & 100.0 & 267 & 100.0 & 395 & 100.0 \\
\hline
\end{tabular}

Tab. 2: Selbstsicht der befragten Studierenden

Self-view of interviewed students

Le point de vue des étudiants interrogés

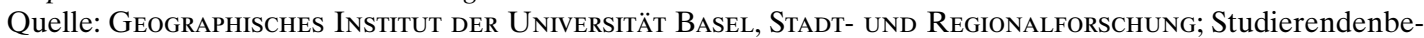
fragung 2009

Teilnahme an Austauschprogrammen während des Studiums eher gering, allerdings hatte fast die Hälfte der Studierenden während ihrer Schulzeit einen Auslandsaufenthalt gemacht. Am mobilsten sind in dieser Hinsicht die Freiburger Studierenden, die deutlich häufiger als ihre Nachbarn an Austauschprogrammen für Schüler und für Studierende teilgenommen hatten.

Welche Verkehrsmittel die Studierenden für ihre Aktivitäten im Nachbarland nutzen, hängt mit der vorhandenen Infrastruktur und mit dem Zweck der Fahrt zusammen. Basler Studierende, die es nicht weit ins Ausland haben, nutzen für Freizeitzwecke häufig das Fahrrad; gehen sie einkaufen oder essen, so benutzen sie eher das Auto. Die öffentlichen Verkehrsmittel wie die Regio-S-Bahn und grenzüberschreitende Buslinien werden ebenfalls häufig genutzt und sind für den Besuch kultureller Veranstaltungen und für Universitätsbesuche die erste Wahl.

Studierende aus Freiburg bevorzugen für Fahrten in die Nordwestschweiz mehrheitlich die öffentlichen Verkehrsmittel, unabhängig vom Zweck der Reise. Ins Elsass gelangen sie dagegen überwiegend mit dem Auto, was angesichts der umständlichen Bus- und Zugverbindungen nicht überrascht. Einzig für den Besuch von universitären Veranstaltungen nutzen sie die öffentlichen Verkehrsmittel. Das gleiche Muster zeigt sich bei den Mulhouser Studierenden, die in die Nordwestschweiz fahren: sie nutzen ebenfalls mehrheitlich die öffentlichen Verkehrsmittel für Universitätsbesuche und ansonsten das Auto. Nach Südbaden gelangen die Studierenden aus Mulhouse dagegen fast ausschliesslich mit dem Auto. In Anbetracht dieser Ergebnisse ist es nicht verwunderlich, dass die Studierenden aus Mulhouse den Öffentlichen Verkehr als trennendes Element in der Regio betrachten.

\subsection{Wahrnehmung der Grenzen und regionale Zugehörigkeit}

Da die Grenzen in der Regio TriRhena einerseits allgegenwärtig und andererseits sehr durchlässig sind, kann es aufschlussreich sein festzustellen, wie die Grenzen von den Befragten wahrgenommen werden. Am häufigsten wurde die sprachliche Barriere genannt, gefolgt von der positiv besetzten Bezeichnung als Vielfalt und Abwechslung. In Freiburg und Mulhouse wurden die Grenzen viel stärker als in Basel als unbedeutendes Relikt aus der Vergangenheit wahrgenommen. Die Basler Studierenden sehen die Grenze dagegen eher als wirtschaftliches Hindernis.

Auf die Frage, wie sie sich selbst am ehesten bezeichnen würden, antwortete knapp die Hälfte der Basler Studierenden, dass sie sich am meisten mit der eigenen Nation verbunden sehen (Tab. 2). Weiter sieht sich ein Viertel der in Basel Studierenden am liebsten als Europäer. Bei der Antwortmöglichkeit «Anderes» wurden vor allem «Weltbürger» und «Basler» genannt. In Freiburg ist das europäische Bewusstsein am ausgeprägtesten. Weniger als ein Drittel der Befragten identifiziert sich mit Deutschland. In Mulhouse kommt das Nationalgefühl, wie in Basel, an erster Stelle. Daneben sieht sich etwa rund ein Viertel der Studierenden der Universität in Mulhouse als ElsässerInnen, und erst dann folgt eine europäische Zuordnung. 
Das viel geringere Nationalgefühl und das grössere europäische Bewusstsein der Freiburger Befragten sind wohl im Zusammenhang mit der Geschichte zu sehen, aufgrund derer viele Deutsche ein distanziertes Verhältnis zum «Nationalstolz» haben. Es muss auch berücksichtigt werden, dass die räumliche Identifikation des Einzelnen vielschichtig aufgebaut ist, und je nach Handlungskontext unterschiedliche Identitäten im Vordergrund stehen können (HARD 1987: 137). Möglicherweise ist eine regionale Identität der Befragten zwar vorhanden, aber eben weniger ausgeprägt als beispielsweise das nationale oder europäische Zugehörigkeitsgefühl. Daher wurden die Studierenden auch gefragt, ob sie ein grenzübergreifendes «Wir-Gefühl» in der Regio wahrnehmen. In Basel ist dieser «RegioGeist» am wenigsten ausgeprägt, gefolgt von Freiburg. In Mulhouse wird ein Zusammengehörigkeitsgefühl über die Grenzen hinweg deutlich am stärksten wahrgenommen.

Identität und Wir-Gefühl zeigen sich auch daran, wenn man sich vorstellen kann, in einem anderen Teil der Regio zu wohnen. Hier scheinen die Studierenden recht offen zu sein: Über zwei Drittel aller Befragten könnten sich vorstellen, jenseits der Grenze zu wohnen. Bei der genaueren Betrachtung ist augenfällig, dass sich die positiven Antworten je nach Herkunft der Befragten erheblich unterscheiden. So sehen mehr als drei Viertel der Freiburger Studierenden das Wohnen jenseits der Grenze als mögliche Option, wohingegen die Basler Studierenden nur gut zur Hälfte dazu bereit wären.

\subsection{Institutionalisierung der grenzüberschreitenden Zusammenarbeit}

Die langjährigen Bemühungen um einen Aufbau der institutionellen Zusammenarbeit scheinen im Bewusstsein der Menschen angekommen zu sein. Die Mehrheit der Studierenden der drei Länder assoziiert mit dem Begriff «Regio TriRhena» eine grenzüberschreitende Zusammenarbeit auf institutionalisierter Ebene. Die Studierenden wurden diesbezüglich gefragt, welche Institutionen sie spontan mit der trinationalen Regio verbinden. Die häufigste Nennung war der Hochschulverbund EUCOR. Wenn man noch Universitäten und allgemeine schulische Zusammenarbeit mit einbezieht, dann bezieht sich ein Fünftel (in Basel) bis die Hälfte (in Freiburg) aller Assoziationen auf den Bereich Bildung. Dass der Bereich Bildung derart prominent vertreten ist, überrascht nicht, angesichts des Fokus der Umfrage auf Studierende. Die Bekanntheit des EUCOR steht allerdings im Gegensatz zum verschwindend geringen Anteil der Studierenden, die an diesem Programm teilnehmen. Die regionale politische Zusammenarbeit, manifestiert z.B. durch Nennungen der Regio TriRhena oder der Oberrheinkonferenz, nimmt in der Schweiz einen höheren Stellenwert ein als in den beiden Nachbarländern. Weitere Nennungen bezogen sich auf den öffentlichen Verkehr (vor allem in Basel genannt), die Kultur, Wirtschaft und den EuroAirport Basel-Mulhouse-Freiburg.

Erwähnenswert ist schliesslich noch die hohe Zahl von Personen, die freie Assoziationen mit höchst unterschiedlichen Institutionen hatten, wie Sportvereine, Medien, Bürgerinitiativen oder Umweltvereine. Dies zeigt, wie facettenreich und persönlich die Assoziationen sind, die sich auf die internationale Region beziehen.

Sämtliche Teilnehmer an der Befragung haben auf die Frage geantwortet, in welchen Bereichen die grenzübergreifende Zusammenarbeit vertieft werden soll. Von einer Mehrheit der Befragten in allen drei Ländern wird eine intensivierte Zusammenarbeit im öffentlichen Verkehr, in der Bildung und im Natur- und Umweltschutz gefordert. In den Bereichen Wirtschaft und Raumplanung halten etwas weniger Studierende eine weitere Vertiefung für wichtig. Der Wunsch nach verstärkter Zusammenarbeit im Bereich Sicherheit rangiert in allen drei Ländern an letzter Stelle.

\section{Diskussion und Fazit}

In der Regio TriRhena sind ausgeprägte grenzüberschreitende Aktivitäten der Bevölkerung beim Einkaufen und in der Freizeit zu beobachten, wobei die Unterschiede im Preisniveau einen materiellen Anreiz darstellen. Dies ist mit der Situation in anderen europäischen Grenzregionen vergleichbar, wo ebenfalls Einkauf und Freizeitaktivitäten die häufigsten Gründe für Grenzüberquerungen darstellen (Fritsche \& Studer 2008: 16; KacZMareK \& StryJaKIEWICZ 2006: 67; Roose 2010: 154; Wiegelmann-Uhlig 2001: 146). Die häufigen Grenzüberquerungen scheinen aber im Allgemeinen nicht $\mathrm{zu}$ intensiven sozialen Kontakten geführt zu haben. Auch dieses Bild deckt sich mit Beobachtungen aus anderen Grenzräumen wie der Grenze zwischen Deutschland und Polen, Tschechien oder den Niederlanden (KACZMAREK \& STRYJAKIEWICZ 2006: 67; STRÜVER 2005: 279; WeIGL 2007: 347).

Eine grenzübergreifende Identifikation der Studierenden in der Regio TriRhena äussert sich deutlich häufiger in der Selbstsicht als Europäer denn als Regiobewohner. $\mathrm{Zu}$ einem vergleichbaren Ergebnis kommt eine Untersuchung von Roose (2010: 219) in den drei deutschen Grenzstädten Frankfurt an der Oder, Saarbrücken und Passau. Auch wenn die Identifikation als Regio-Bewohner für die meisten Befragten nicht an erster Stelle steht, nehmen doch viele Studierende zumindest ein schwaches grenzüberschreitendes «Wir- 
Gefühl» wahr, und eine deutliche Mehrheit von ihnen kann sich vorstellen, in einem anderen Teil der Regio zu wohnen.

Die Mehrzahl der Studierenden befürwortet eine vertiefte grenzüberschreitende Zusammenarbeit der Institutionen, vor allem in den Bereichen Bildung, öffentlicher Verkehr und Natur- und Umweltschutz. Hier ist der Vergleich mit anderen europäischen Grenzräumen weniger eindeutig. Während beispielsweise die Bevölkerung im Bodenseeraum dem Ausbau des grenzüberschreitenden öffentlichen Verkehrs ebenfalls eine hohe Priorität einräumt (Fritsche \& StUder 2008: 43), wurde 2006 in Frankfurt an der Oder der Bau einer Strassenbahn in die polnische Nachbarstadt Słubice in einer Bürgerbefragung massiv abgelehnt. Im Unterschied zu den jüngeren Euroregionen in Mittel- und Osteuropa spielen Sicherheitsfragen in der Regio TriRhena nur eine marginale Rolle, die Menschen scheinen sich trotz der Grenzlage sicher zu fühlen.

Die Studierenden in der Regio TriRhena nehmen die Angebote von EUCOR zum Austausch mit den Nachbaruniversitäten nur in geringem Masse wahr. Allgemein werden die Austauschprogramme und andere institutionalisierte Angebote grenzüberschreitender Aktivitäten weniger genutzt als die Möglichkeiten, relativ spontan in die angrenzenden Regionen zu fahren, um dort eher «alltäglichen» Aktivitäten nachzugehen wie einkaufen, essen oder sich erholen. Das Mobilitäts- und Konsumverhalten der Studierenden kann einerseits als Ausdruck eines starken regionalen Bewusstseins im Sinne einer freien Mobilität in den Teilräumen interpretiert werden, andererseits kann es auch als rein zweckrationales Handeln gesehen werden, das nicht mit einem Bewusstseinswandel der Beteiligten einhergeht.

Möchte man, wie es sich vielfältige Institutionen und Kooperationen am Oberrhein zum Ziel gesetzt haben, die Interaktion und gemeinsame Identität in der Regio TriRhena fördern, so lassen sich aus der vorliegenden Studie mehrere Ansatzpunkte ableiten. Die Studierenden fordern eine engere Zusammenarbeit im Bildungsbereich, obwohl hier schon diverse Projekte bestehen. Möglicherweise werden die bestehenden Programme nicht ausreichend kommuniziert oder in die herkömmlichen Angebote der Universitäten eingebunden. Eine verbesserte Zusammenarbeit im öffentlichen Verkehr, im Natur- und Umweltschutz und in der Wirtschaft sind weitere zentrale Forderungen. Gerade in den Verkehrsverbindungen zwischen Südbaden und Elsass besteht noch ein grosses Potential, die Region enger zusammenrücken zu lassen. Die Überwindung der sprachlichen Barrieren zwischen dem Elsass einerseits und der Nordwestschweiz und Südbaden andererseits könnte die Kommunikation und soziale Interaktion - und damit eine der Grundvoraussetzungen für eine gemeinsame Identität - erheblich verbessern.

\section{Literaturverzeichnis}

Aschauer, W. (2000): Regionale Identität als empirischer Untersuchungsgegenstand - Aufbruch in die «Normalwissenschaft»? - In: Geographische Revue 2, 1:55-60.

Blotevogel, H., Heinritz, G. \& H. Popp (1989): «Regionalbewusstsein». Zum Stand der Diskussion um einen Stein des Anstosses. - In: Geographische Zeitschrift 77, 2: 65-88.

Eder SANDTner, S. \& M. SANDTner (2003): Regionale Identität über die Grenzen? Die Regio TriRhena im Bewusstsein der Bevölkerung. - In: SCHNEIDER-SLIWA, R. (Hrsg.): Regio TriRhena und südlicher Oberrhein - ein Raum ohne Grenzen? - = Basler Stadt- und Regionalforschung 22, Basel: 7-17.

Fichter-Wolf, H. (2009): Hochschulmilieus in Grenzräumen - Impulsgeber einer Koevolution von Raum und Wissen. - In: Matthiesen, U. \& G. Mahnken (Hrsg.): Das Wissen der Städte. Neue stadtregionale Entwicklungsdynamiken im Kontext von Wissen, Milieus und Governance. - Wiesbaden: VS Verlag für Sozialwissenschaften: 201-217.

FichtNER, U. (1988): Regionale Identität am südlichen Oberrhein - zur Leistungsfähigkeit eines verhaltenstheoretischen Ansatzes. - In: Berichte zur deutschen Landeskunde 62, 1: 109-139.

Fritsche, A. \& L. StUder (2008): Grenzüberschreitung in der Bodenseeregion. Durch Partizipation vom «ihr» zum «wir»? - = Veröffentlichungen des Instituts für sozialwissenschaftliche Regionalforschung 6, Bregenz: $1-52$.

Guichonnet, P. \& C. RAFFestin (1974): Géographie des frontières. - Vendôme (France): Presses Universitaires de France.

HARD, G. (1987): «Bewusstseinräume». Interpretationen zu geographischen Versuchen, regionales Bewusstsein zu erforschen. - In: Geographische Zeitschrift 75, 3: 127-148.

HartweG, F. (2003): Die Sprachen im Elsass. Banalisierung, Rückzug in die ökologische Nische oder Öffnung nach Europa? - In: ReICH, H.H. (Hrsg.): Zwischen Regionen. Grenzüberschreitende Beziehungen am Beispiel des Oberrheins. - = Landauer Schriften zur Kommunikations- und Kulturwissenschaft 2, Landau: Knecht Verlag: 125-150.

IPSEN, D. (1994): Regionale Identität. Überlegungen zum politischen Charakter einer psychosozialen Raumkategorie. - In: LindNER, R. (Hrsg.): Die Wiederkehr des Regionalen. Über neue Formen kultureller Identität. - Frankfurt am Main: Campus Verlag: 232-254.

Kaczmarek, T. \& T. StryjaKiewicz (2006): Grenzüberschreitende Entwicklung und Kooperation im 
deutsch-polnischen Grenzraum aus polnischer Sicht. - In: Europa Regional 14, 2: 61-70.

LEIMGRUBER, W. (2005): Boundaries and transborder relations, or the hole in the prison wall: On the necessity of superfluous limits and boundaries. - In: GeoJournal 64, 3: 239-248.

Martinez, O.J. (1994): The dynamics of border interaction. New approaches to border analysis. - In: ScHOFIELD, C.H. (Hrsg.): Global boundaries. $-=$ World Boundaries 1, London: 1-15.

MüHLER, K. \& K. Opp (2004): Region und Nation. Zu den Ursachen und Wirkungen regionaler und überregionaler Identifikation. - Wiesbaden: VS Verlag für Sozialwissenschaften.

MÜHLER, K. \& K. OpP (2008): Ursachen für die Identifikation von Bürgern mit ihrer Region und Wirkungen auf ihr individuelles Handeln. - http://www.kulturregionen.org/2008_symposium/muehler-opp_zusammenfassung_regionale_Identifikation.pdf 4.12.2009.

ReITEL, B. (2007): Les agglomérations transfrontalières: des systèmes urbains en voie d'intégration? Les espaces urbains de la «frontière» du territoire français. - In: Geographica Helvetica 62, 1: 5-15.

Roose, J. (2010): Vergesellschaftung an Europas Binnengrenzen. Eine vergleichende Studie zu den Bedingungen sozialer Integration. - Wiesbaden: VS Verlag für Sozialwissenschaften.

Schneider, C., Franze, M. \& K. Daumenlang (2003): Psychologische Aspekte der Interregionalität. Ein Beitrag zu einer «Theorie der Interregionalität». - In: ReIch, H.H. (Hrsg.): Zwischen Regionen. Grenzüberschreitende Beziehungen am Beispiel des Oberrheins. - = Landauer Schriften zur Kommunikations- und Kulturwissenschaft 2, Landau: Knecht Verlag: 111-124.

Schneider-Sliwa, R., Erismann, C., Saalfrank, C. \& C. Griebel (2009): Regionale Identität in der Regio TriRhena. Wahrnehmung von Studierenden der Universitäten Basel, Freiburg i.Br. und Mulhouse. - = Basler Stadt- und Regionalforschung 32, Basel.

Schuhbauer, J. (1996): Wirtschaftsbezogene regionale Identität. - = Mannheimer Geographische Arbeiten 42, Mannheim.

SimmeL, G. (1908; 1992): Der Raum und die örtliche Ordnung der Gesellschaft. - Bd. XI, Simmel-Gesamtausgabe, Frankfurt am Main: Suhrkamp.

STRÜVER, A. (2005): Grenzen der Grenzüberschreitung: Deutsch-niederländische Beziehungen in ihrer Ambivalenz aus Nähe und Distanz. - In: Berichte zur deutschen Landeskunde 79, 2-3: 277-289.

WeIGL, M. (2005): Grenze als Identitätsstiftung in der Gegenwart. - http://www.identityresearch.eu/raum/ Grenze.pdf 26.11.2009.

WeIGL, M. (2007): Die identitätsstiftende Wirkung der bayerisch-böhmischen Grenze in der Gegenwart. - In: Luft, R. \& L. Eiber (Hrsg.): Bayern und Böhmen. Kontakt, Konflikt, Kultur. - =Veröffentlichungen des Collegium Carolinum 111, München: 333-360.
WERLEN, B. (1992): Regionale oder kulturelle Identität? Eine Problemskizze. - In: Berichte zur deutschen Landeskunde 66, 1:9-32.

Wiegelmann-Uhlig, E. (2001): Sozioökonomische Verflechtungen im Raum PAMINA. - In: GeIger, M. (Hrsg.): PAMINA - Europäische Region mit Zukunft. Baden, Elsass und Pfalz in grenzüberschreitender Kooperation. - Speyer: Verlag der Pfälzischen Gesellschaft zur Förderung der Wissenschaften: 144-160.

\section{Zusammenfassung: Grenzüberschreitende regionale Identität in der Regio TriRhena von Studierenden der Universitäten Basel, Freiburg im Breisgau und Mulhouse}

In der trinationalen Region am Oberrhein bilden die politische Zusammenarbeit und die wirtschaftlichen Verbindungen zwischen den Teilräumen Nordwestschweiz, Elsass und Südbaden gute Voraussetzungen für eine gemeinsame, grenzüberschreitende regionale Identität. In einer Befragung von Studierenden an drei Universitäten in der Regio TriRhena wurden die raumbezogene Identifikation der Studierenden, ihre grenzüberschreitenden sozialen Kontakte und ihre Mobilität untersucht. Die Mehrheit der Studierenden rückt die nationale oder europäische Identität in den Vordergrund, während die Identität als «Regio-Bewohner/in» nur eine untergeordnete Rolle spielt. Die Mobilität der Befragten in die Nachbarregionen ist dagegen sehr ausgeprägt, besonders für Einkäufe und Freizeitaktivitäten. Grenzüberschreitende regionale Identität kann also vor allem im Hinblick auf das raumrelevante Handeln der Studierenden ausgemacht werden und weniger in Bezug auf die Artikulation von regionaler Zugehörigkeit.

Schlüsselwörter: Südlicher Oberrhein, raumbezogene Identität, Grenzregion, grenzüberschreitende Mobilität, grenzüberschreitende Kooperation, trinationale Region Basel

\section{Summary: Crossborder regional identity in the TriRhena region of university students in Basel, Freiburg im Breisgau and Mulhouse}

In the trinational region of the Upper Rhine valley, political cooperation and economic links between the subregions Northwest Switzerland, Alsace and South Baden provide suitable conditions for a common, crossborder identity. Students at three universities in the TriRhena region were asked about their spatial identity, crossborder social contacts and mobility. The majority of students identified themselves stronger with their national or European identity than with their status as residents of the TriRhena region. Crossborder mobility of interviewees is, however, significant, particularly concerning shopping and entertainment. Crossborder regional identity may thus be seen more in spatial activities of students than in the articulation of regional affiliation. 
Keywords: Southern Upper Rhine, spatial identification, border region, crossborder mobility, crossborder cooperation, trinational region Basel

\section{Résumé: Identité transfrontalière régionale dans la Regio TriRhena. Le point de vue des étudiants des} universités de Bâle, Fribourg-en-Brisgau et Mulhouse Dans la région trinationale du Rhin supérieur, les coopérations politiques et les interactions socio-économiques entre la Suisse du Nord-Ouest, l'Alsace et le Bade méridional forment un contexte favorable à l'émergence d'une identité transfrontalière commune. Dans ce contexte, l'article étudie l'identification spatiale des étudiants de trois universités de la Regio TriRhena, leurs liens sociaux transfrontaliers ainsi que leur mobilité. Les résultats montrent que la majorité des étudiants se réfèrent prioritairement à une identité nationale ou européenne, tandis que l'identité transfrontalière de la Regio ne joue qu'un rôle mineur. La mobilité des personnes interrogées en direction des régions voisines est cependant élevée, surtout en matière d'achats et de loisirs. L'identité transfrontalière régionale peut alors être identifiée selon l'espace d'action des étudiants concernés, plutôt qu'en termes d'appartenance régionale commune.

Mots-clés: sud du Rhin supérieur, identité territoriale, région frontalière, mobilité transfrontalière, coopération transfrontalière, région trinationale de Bâle

Christine Griebel, M.A., Geographisches Institut der Universität Basel, Humangeographie / Stadt- und Regionalforschung, Klingelbergstrasse 27, CH-4056 Basel, Schweiz.

e-mail: christine.griebel@unibas.ch

\section{Manuskripteingang/received/manuscrit entré le}

15.1.2010

Annahme zum Druck/accepted for publication/accepté pour l'impression: 15.3 .2010 